\title{
When slower is faster
}

\author{
Carlos Gershenson ${ }^{1,2,3,4,5}$ and Dirk Helbing ${ }^{6}$ \\ ${ }^{1}$ Instituto de Investigaciones en Matemáticas Aplicadas y en Sistemas, \\ Universidad Nacional Autónoma de México. \\ cgg@unam.mx http://turing.iimas.unam.mx/ cgg \\ ${ }^{2}$ Centro de Ciencias de la Complejidad, UNAM, México. \\ 3 SENSEable City Lab, Massachusetts Institute of Technology, USA. \\ ${ }^{4}$ MoBS Lab, Northeastern University, USA. \\ ${ }^{5}$ ITMO University, St. Petersburg, Russian Federation. \\ ${ }^{6}$ Department of Humanities, Social and Political Sciences (GESS) \\ ETH Zürich \\ dirk.helbing@gess.ethz.ch http://www.coss.ethz.ch
}

October 21, 2015

\begin{abstract}
The slower is faster (SIF) effect occurs when a system performs worse as its components try to do better. Thus, a moderate individual efficiency actually leads to a better systemic performance. The SIF effect takes place in a variety of phenomena. We review studies and examples of the SIF effect in pedestrian dynamics, vehicle traffic, traffic light control, logistics, public transport, social dynamics, ecological systems, and adaptation. Drawing on these examples, we generalize common features of the SIF effect and suggest possible future lines of research.
\end{abstract}

\section{Introduction}

How fast should an athlete run a race? If she goes too fast, she will burn out and become tired before finishing. If she runs conservatively, she will not get tired, but will not make her best time. To minimize her race time, she has to go as fast as possible but without burning out. If she goes faster, she will actually race more slowly. This is an example of the "slower-is-faster" (SIF) effect: in order to run faster, sometimes it is necessary to run slower, not to burn out. It is not trivial to calculate the running speed which will lead to the best race, as this depends on the athlete, race distance, track, temperature, humidity, and daily performance: Running $100 \mathrm{~m}$ dash should be done as fast as you can, while running a marathon demands a carefully paced race. How fast would an athlete run a marathon if she started with a speed for a $100 \mathrm{~m}$ ? To finish the marathon successfully, she would obviously have to run more slowly. 
There are several other examples of the SIF effect, which will be described in the next section. We then generalize the common features of these phenomena to discuss potential causes and promising lines of research towards a unified explanation of the SIF effect.

\section{Examples}

\subsection{Pedestrian evacuation}

Perhaps the first formal study of the SIF effect was related to pedestrian flows (Helbing et al., 2000a). Modelling crowds like self-driven particles with "social forces" interacting among them (Helbing and Molnár, 1995; Helbing et al., 2000b), it has been shown that when individuals try to evacuate a room too quickly, they lead to intermittent clogging and a reduced outflow as compared to a calmer evacuation. In this context, the SIF effect is also known as "freezing by heating" (Stanley, 2000). Trying to exit fast makes pedestrians slower, while calmer people manage to exit faster. This has led people to suggest obstacles close to exits, precisely to reduce friction (Helbing et al., 2005; Heliövaara et al., 2013). Counterintuitively, a slowdown of the evacuation can increase the outflow. Also, in a related study of aircraft evaluation, it was found that there is a critical door width which determines whether "competitive" evacuation will increase or decrease evacuation time (Kirchner et al., 2003). In other words, pushy people will evacuate slower if there are narrow doors (SIF), but will evacuate faster if the doors are wide enough (FIF, faster-is-faster).

\subsection{Pedestrians crossing a road}

Another example concerns mixed pedestrian and vehicle traffic. Imagine pedestrians are trying to cross a road at a location where there is no traffic light and no pedestrian crossing is marked. This is a typical situation along speed-reduced roads (e.g. with a speed limit of $30 \mathrm{~km} / \mathrm{h}$ ) or in shared spaces for multi-modal use. Pedestrians would cross when the gap between two successive vehicles exceeds a certain critical separation that ensures a safe crossing of the road. However, there are two types of pedestrians: patient and pushy ones. Pushy pedestrians might force a vehicle to slow down while patient pedestrians would not do this, i.e. they would wait for a larger gap. Surprisingly, if all pedestrians were of the patient type, on average they would have to wait for a shorter time period (Jiang et al., 2006).

How does this SIF effect come about? When a pushy pedestrian has slowed a vehicle down, other arriving pedestrians will pass the road, too, and it takes a long time until no further pedestrians arrive, and the stopped cars can accelerate again. During the waiting time, however, a long vehicle queue has formed, such that no large enough gap to cross the road occurs between vehicles until the entire vehicle queue has dissolved. As a consequence, pedestrians will have to wait for a long time until they can cross again. Altogether, it is better if pedestrians wait for large enough gaps such that they don't force vehicles to slow down. 


\subsection{Vehicle traffic}

SIF effects are also known from vehicle traffic (Helbing and Huberman, 1998; Helbing and Treiber, 1998; Helbing, 2001; Helbing and Nagel, 2004). Surprisingly, speed limits can sometimes reduce travel times. This is the case, when the traffic density enters the multi-stable ("metastable") regime. Then, traffic flow is sensitive to disruptions and may break down, which causes largely increased travel times. A speed limit can delay the breakdown of fluid traffic flows, because it reduces the variability of vehicle speeds. This homogenization avoids disturbances in the flow, which are big enough to trigger a breakdown (i.e. have a "super-critical" amplitude).

If vehicles go fast, the safety distance between vehicles must be increased. Thus, less vehicles will be able to use a road. For example, at $80 \mathrm{~km} / \mathrm{h}$, a maximum capacity of about 22 vehicles per $\mathrm{km}$ per lane is reached before free traffic flow breaks down. At $120 \mathrm{~km} / \mathrm{h}$, this capacity is reduced to about 15 vehicles per $\mathrm{km}$ per lane. Once vehicles slow down due to an increased density, traffic jams will propagate, as a following car tends to brake more than the vehicle ahead. This phase transition of "stable" to "unstable" flow in traffic depends on the desired speed. Thus, to maximize flow, the optimal speed of a highway will depend on the current density. However, the maximum flow lies at the tipping point, and thus a small perturbation can trigger stop-and-go waves which can reduce the highway capacity by $30 \%$.

A similar consideration applies to over-taking maneuvers (Kesting et al., 2007). Pushy drivers might force cars in the neighboring lane to slow down when changing lanes to overtake another car, while patient drivers would not do this. As a consequence, pushy drivers may cause a disruption of metastable traffic flow, which may trigger a breakdown ("capacity drop"). Consequently, patient drivers will avoid or delay a breakdown of traffic flow, thereby managing to progress faster on average. One may also formulate this in game theoretical terms. When traffic flow is metastable, drivers are faced with a social dilemma situation: choosing a patient behavior will be beneficial for everyone, while pushy behavior will produce small individual advantages at the cost of other drivers. As a consequence, a "tragedy of the commons" results: pushy drivers undermine the stability of the metastable traffic flow, causing congestion that forces everyone to spend more time on travel.

A complementary phenomenon is observed in Braess's paradox (Braess et al., 2005; Steinberg and Zang 1983), where adding roads can reduce the flow capacity of a road network.

\subsection{Traffic light control}

The SIF effect is also found in further systems such as urban traffic light control (Helbing and Mazloumian, 2009). Here, a first-come-first-serve approach works only well at low traffic volumes. Otherwise, forcing vehicles to wait for some time can speed up their overall progress. The reason is that this will produce vehicle platoons, such that a green light will efficiently serve many vehicles in a short time period (Gershenson, 2005; Gershenson and Rosenblueth, 2012; Zubillaga et al., 2014). Similarly, it may be better to switch traffic lights less frequently, because switching reduces service times (due to time lost on amber lights). A "green wave", i.e., a coordination of vehicle flows such that several successive traffic lights can be passed without stopping, is another good example demonstrating that waiting (at a red light) may be rewarding altogether. 
Similarly interesting observations can be made for self-organized traffic light control ("self-control"), which based on decentralized flow control ("distributed control") (Lämmer et al., 2006; Lämmer and Helbing, 2008, 2010; Helbing, 2013). If each intersection strictly minimizes the travel times of all vehicles approaching it, according to the principle of a "homo economicus", this can create efficient traffic flows, when these are low or moderate ("invisible hand phenomenon"). However, vehicle queues might get out of hand when the intersection utilization increases. Therefore, it is beneficial to interrupt travel time minimization in order to clear a vehicle queue when it has grown beyond a certain "critical" limit. This avoids spillover effects, which would block other intersections and cause a quick spreading of congestion over large parts of a city. Consequently, waiting for a long queue to be cleared can speed up traffic altogether. Putting it differently, other-regarding self-organization can beat the selfish optimization à la "homo economicus", who strictly does the best, but neglects a coordination with neighbors.

\subsection{Logistics and supply chains}

Similar phenomena as in urban traffic flows are found in logistic systems and supply chains (Helbing and Lär 2005; Helbing et al., 2006; Seidel et al., 2008; Peters et al., 2008). We have studied, for example, a case of harbor logistics using automated guided vehicles for container transport. Our proposal was to reduce the speed of these vehicles. This reduced the required safety distances between vehicles, such that less conflicts of movement occurred, and the automatic guided vehicles had to wait less. In this way, transportation times could be overall reduced, even though movement times obviously increased.

We made a similar observation in semiconductor production. So-called "wet benches" are used to etch structures into silicium wavers, using particular chemical solutions. To achieve good results, the wavers should stay in the chemical baths longer than a minimum and shorter than a maximum time period. Therefore, it might happen that several silicium wavers need to be moved around at about the same time, while a moving gripper, the "handler", must make sure to stay within the minimum and maximum times. It turns out that slightly extending the exposure time in the chemical bathes enables much better coordination of the movement processes, thereby reaching a 30 percent higher throughput.

In a third logistics project, the throughput of a packaging plant had to be increased. One of the central production machines of this plant frequently broke down, such that it was operated at full speed whenever it was operating well. However, this filled the buffer of the production plant to an extent that made its operation inefficient. This effect can be understood with queuing theory, according to which cycle times can dramatically increase as the capacity of a buffer is approached.

\subsection{Public transport}

In public transportation systems, it is desirable to have equal headways between vehicles such as buses, i.e., to reach regular time separations between vehicles. However, the equal headway configuration is unstable (Gershenson and Pineda, 2009). Forcing equal headways minimizes waiting times at stations. Nevertheless, the travel time is not independent of the waiting time, as equal headways imply idling or leaving some passengers at stations. This is 
because there is a different demand for each vehicle at each station. Still, self-organization can be used to regulate the headways adaptively (Gershenson, 2011a). Considering only local information, vehicles are able to respond adaptively to the immediate demand of each station. With this method, there is also a SIF effect, as passengers wait more time at a station, but reach their destination faster once they board a vehicle because there is no idling necessary to maintain equal headways.

\subsection{Social dynamics}

Axelrod (Axelrod, 1997) proposed an interesting model of opinion formation. In this model, agents may change their opinion depending on the opinion of their neighbors. Eventually, the opinions converge to a stable state. However, if agents switch their opinion too fast, this might delay convergence (Stark et al., 2008a,b). Thus, there is a SIF effect because the fastest convergence will not necessarily be obtained with the fastest opinion change. In this model, there is also a phase transition which is probably related to the optimal opinion change rate (Vilone et al., 2002). There is also experimental evidence of the SIF effect in group decisions. While designing new buildings, slowing down the deliberative process of teams accelerates the design and construction of buildings (Cross et al., 2015).

Extrapolating these results, one may speculate that high-frequency financial trading (Narang, 2013) may also produce a SIF effect, in the sense that trading at the microseconds scale generates price and information fluctuations which could generate market instabilities leading to crashes and slower economic growth (Easley et al., 2011).

In combinatorial game theory (Siegel, 2013), sometimes the best possible move (e.g. taking a queen in chess) is not necessarily the best move in the long term. In other words, having the highest possible gain at each move does not give necessarily the best game result (Russell and Norvig, 2003, pp. 163-171).

\subsection{Ecology}

If a predator consumes its prey too fast, there will be no prey to consume and the predator population will decline. Thus, a "prudent predator" (Slobodkin, 1961; Goodnight et al., 2008) will actually spread faster than a greedy one. A similar SIF effect applies to parasite/host relationships, where parasites taking too many resources from their host are causing their own demise (Dunne et al., 2013). Over long timescales, evolution will favor symbiotic over parasitic relationships, promoting mechanisms for cooperation which can regulate the interaction between different individuals (Sachs et al., 2004; Virgo et al., 2013).

We can see that the same principle applies to natural resource management, such as fisheries (Pauly et al., 1998). If catches are excessive, there will not be enough fishes left to maintain their numbers, and subsequent catches will be poor. It is estimated that apart from its ecological impact, overfishing has left a void of US\$32 billion per year due to reduced catches (Toppe et al., 2012). However, regulating how much fish is caught per year is complicated. The maximum sustainable yield varies from species to species (Maunder, 2002), so the calculation of the "optimal" yields per year is not at all a trivial task. 


\subsection{Adaptation}

Evolution, development, and learning can be seen as different types of adaptation, acting at different timescales (Aguilar et al., 2014). Also, adaptation can be seen as a type of search (Downing, 2015). In computational searches, it is known that there needs to be a balance between "exploration" and "exploitation" (Blum and Roli, 2003). An algorithm can explore different possible solutions or exploit solutions similar to those already found. Too much exploration or too much exploitation will lead to longer search times. Too much breadth (exploration) will only explore slightly different types of solution, while too much depth (exploitation) might lead to local optima and data overfitting. A key problem is that the precise balance between exploration (diversification) and exploitation (intensification) depends the precise search space (Wolpert and Macready, 1995, 1997) and timescale (Gershenson, 2010; Watson et al., 2011).

An example of the SIF was described in biological evolution (Sellis et al., 2011). Haploid species (with a single copy of their genome, such as bacteria) can adapt faster than diploid species (with two copies of their genome, such as most plants and animals). Still, in a fastchanging environment, haploids adapt "too fast", i.e. the population loses genome variation, while diploids can maintain a diversity. Having such a diversity, diploids can adapt faster to changes in their environment, as they can begin an evolutionary search from many different states at once.

In principle, it would be desirable to find a solution as fast as possible, exploiting current solutions. Still, as mentioned, this might lead to suboptimality (SIF) in evolving new features, optimizing a multidimensional function, or training a neural network. To be efficient, search should eventually "slow down", as it is known from "simulated annealing". As too much exploration would be suboptimal also, the critical question is how to find the precise balance to speed up search as much as possible. Computationally, it seems that this question is not reducible (Wolfram, 2002), so we can only know a posteriori the precise balance for a given problem. Still, finding this balance would be necessary for adiabatic quantum computation (Farhi et al., 2000; Aharonov et al., 2008), as if the system evolves too fast, the information is destroyed.

\section{Generalization}

What do all the above examples have in common? They can be described as complex dynamical systems composed of many non-linearly interacting components. In the above cases, the system can have at least two different states: an efficient and an inefficient one. Unfortunately, the efficient state can be unstable, such that the system will tend to end up in the inefficient state. In the case of freeway traffic, for example, it is well known that the most efficient state (with the highest throughput) is unstable, thereby causing the traffic flow to break down sooner or later ("capacity drop"). To avoid the undesired outcome, the system components must stay sufficiently away from the instability point, which requires them to be somewhat slower than they could be, but as a reward they will be able to sustain a relatively high speed for a long time. If they go faster, the efficient state will break down and trigger another one that is typically slower. This situation might be characterized as a 
"tragedy of the commons" (Hardin, 1968).

Even though it might be counterintuitive, the SIF effect occurs in a broad variety of systems. For practical purposes, many systems have a monotonic relation between "inputs" and "outputs". This is true for systems that "break" (Ashby, 1947). For example, if temperature is increased in a constrained gas with a constant volume, pressure rises. Yet, if temperature increases too much, then the gas container will break, leading to a pressure reduction. Still, without breaking, many physical and non-physical systems have thresholds, where they become unstable and a phase transition to a different systems state occurs. A typical situation of many-particle systems is that they may get overloaded and turn dysfunctional through a cascading effect.

To reduce the SIF effect we can seek to adjust the interactions which cause a reduction in the system performance (Gershenson, 2011b). The vehicle traffic case offers an interesting example: when vehicles go too fast (and their density crosses a critical density), their changes in speed will affect other vehicles, generating an amplification of oscillations, which lead to stop-and-go traffic and, as a consequence, to a reduced average speed. If vehicles go slower, then such oscillations can be avoided and the average speed will be higher. The key here is that the critical speed where traffic flow changes from "laminar" (where FIF) to "unstable" (where SIF) changes with the density. However, suitably designed adaptive systems, such as driver assistance systems, can be used to drive systems towards their best possible performance in their respective context (Gershenson, 2007; Helbing, 2015).

\section{Discussion}

It could be argued that the SIF effect is overly simplistic, as there is only the requirement of having two dynamical phases, where one comes with a reduced efficiency after crossing the phase transition point. Still, as we have presented, the SIF effect shows up in a variety of interesting phenomena at different scales. Thus, we can say that having a better understanding of the SIF effect can be useful and potentially have a broad impact. A challenge lies in characterizing the nature of the different types of interactions which will reduce efficiency (Gershenson, 2013).

We can identify the following necessary conditions for the SIF effect:

1. There is an instability (internal or external) in the system.

2. The instability is amplified, sometimes through cascading effects.

3. There is a transition from the unstable to a new stable state which leads to inefficiency.

4. Such a state can be characterized as "overloaded".

It is worth noting that in some cases, single variables may be stable to perturbations, but their interactions are the ones that trigger instability. This implies that the SIF in these cases has to be studied at two scales: the scale of the components and the scale of the system, as studying components in isolation will not provide enough information to reproduce the SIF effect. 
Whether all phenomena with a SIF effect can be described with the same mathematical framework remains to be seen. We believe this is an avenue of research worth pursuing and with relevant implications for the understanding of complex systems.

\section{Acknowledgments}

We should like to thank Luis Álvarez de Icaza, Jeni Cross, Tom Froese, Marios Kyriazis, Gleb Oshanin, Sui Phang, Frank Schweitzer, Diamantis Sellis, Simone Severini, Thomas Wisdom, Héctor Zenil, and two anonymous referees for useful comments. C.G. was supported by CONACYT projects 212802, 221341, and SNI membership 47907. D.H. was supported by ERC Advanced Grant MOMENTUM 324247.

\section{References}

Aguilar, W., Santamaría Bonfil, G., Froese, T., and Gershenson, C. (2014). The past, present, and future of artificial life. Frontiers in Robotics and AI 1 (8). URL http://dx.doi.org/10.3389/frobt.2014.00008.

Aharonov, D., Van Dam, W., Kempe, J., Landau, Z., Lloyd, S., and Regev, O. (2008). Adiabatic quantum computation is equivalent to standard quantum computation. SIAM review 50 (4): 755-787. URL http://dx.doi.org/10.1137/080734479.

Ashby, W. R. (1947). The nervous system as physical machine: With special reference to the origin of adaptive behavior. Mind 56 (221) (January): 44-59. URL http://tinyurl.com/aqcmdy.

Axelrod, R. (1997). The dissemination of culture: A model with local convergence and global polarization. Journal of Conflict Resolution 41 (2): 203-226. URL http://jcr. sagepub.com/content/41/2/203.abstract.

Blum, C. And Roli, A. (2003). Metaheuristics in combinatorial optimization: Overview and conceptual comparison. ACM Comput. Surv. 35 (3) (Sept.): 268-308. URL http://doi.acm.org/10.1145/937503.937505.

Braess, D., Nagurney, A., And Wakolbinger, T. (2005). On a paradox of traffic planning. Transportation Science 39 (4) (November): 446-450. Translated from the original German: Braess, Dietrich. 1968. Über ein Paradoxon aus der Verkehrsplanung. Unternehmensforschung 12 258-268. URL http://dx.doi.org/10.1287/trsc.1050.0127.

Cross, J. E., Barr, S. K., Putnam, R., Dunbar, B. H., and Plaut, J. M. (2015). The social network of integrative design. Tech. rep., Institute for the Built Environment, Colorado State University, Fort Collins, CO, USA.

Downing, K. L. (2015). Intelligence Emerging: Adaptivity and Search in Evolving Neural Systems. MIT Press, Cambridge, MA, USA. 
Dunne, J. A., Lafferty, K. D., Dobson, A. P., Hechinger, R. F., Kuris, A. M., Martinez, N. D., Mclaughlin, J. P., Mouritsen, K. N., Poulin, R., Reise, K., Stouffer, D. B., Thieltges, D. W., Williams, R. J., And ZANDER, C. D. (2013). Parasites affect food web structure primarily through increased diversity and complexity. PLoS Biol 11 (6) (06): e1001579. URL http://dx.doi.org/10.1371/journal.pbio.1001579.

Easley, D., De Prado, M. M. L., And O'Hara, M. (2011). The microstructure of the "flash crash": Flow toxicity, liquidity crashes, and the probability of informed trading. The Journal of Portfolio Management 37 (2) (Winter): 118-128. URL http://dx.doi.org/10.3905/jpm.2011.37.2.118.

Farhi, E., Goldstone, J., Gutmann, S., And Sipser, M. (2000). Quantum computation by adiabatic evolution. Tech. Rep. CTP-2936, MIT. URL http://arxiv.org/abs/quant-ph/0001106.

Gershenson, C. (2005). Self-organizing traffic lights. Complex Systems 16 (1): 29-53. URL http://www. complex-systems.com/pdf/16-1-2.pdf.

Gershenson, C. (2007). Design and Control of Self-organizing Systems. CopIt Arxives, Mexico. http://tinyurl.com/DCSOS2007. URL http://tinyurl.com/DCSOS2007.

Gershenson, C. (2010). Computing networks: A general framework to contrast neural and swarm cognitions. Paladyn, Journal of Behavioral Robotics 1 (2): 147-153. URL http://dx.doi.org/10.2478/s13230-010-0015-z.

Gershenson, C. (2011a). Self-organization leads to supraoptimal performance in public transportation systems. PLoS ONE 6 (6): e21469. URL http://dx.doi.org/10.1371/journal.pone.0021469.

Gershenson, C. (2011b). The sigma profile: A formal tool to study organization and its evolution at multiple scales. Complexity 16 (5): 37-44. URL http://arxiv.org/abs/0809.0504.

Gershenson, C. (2013). The implications of interactions for science and philosophy. Foundations of Science 18 (4): 781-790. URL http://arxiv.org/abs/1105.2827.

Gershenson, C. And Pineda, L. A. (2009). Why does public transport not arrive on time? The pervasiveness of equal headway instability. PLoS ONE 4 (10): e7292. URL http://dx.doi.org/10.1371/journal. pone.0007292.

Gershenson, C. And Rosenblueth, D. A. (2012). Self-organizing traffic lights at multiple-street intersections. Complexity 17 (4): 23-39. URL http://dx.doi.org/10.1002/cplx.20392.

Goodnight, C., Rauch, E., Sayama, H., De Aguiar, M. A. M., Baranger, M., AND BAR-YAM, Y. (2008). Evolution in spatial predator-prey models and the "prudent predator": The inadequacy of steady-state organism fitness and the 
concept of individual and group selection. Complexity 13 (5): 23-44. URL http://dx.doi.org/10.1002/cplx.20209.

Hardin, G. (1968). The tragedy of the commons. Science 162 (3859): 1243-1248. URL http: //www.sciencemag.org/content/162/3859/1243.abstract.

Helbing, D. (2001). Traffic and related self-driven many-particle systems. Reviews of modern physics $\mathbf{7 3}$ (4): 1067.

HeLbing, D. (2013). Economics 2.0: the natural step towards a self-regulating, participatory market society. Evolutionary and Institutional Economics Review 10 (1): 3-41.

Helbing, D. (2015). Thinking Ahead-Essays on Big Data, Digital Revolution, and Participatory Market Society. Springer.

Helbing, D., Buzna, L., Johansson, A., And Werner, T. (2005). Self-organized pedestrian crowd dynamics: Experiments, simulations, and design solutions. Transportation science 39 (1): 1-24.

Helbing, D., Farkas, I., And Vicsek, T. (2000a). Simulating dynamical features of escape panic. Nature 407 (6803): 487-490.

Helbing, D., Farkas, I. J., And Vicsek, T. (2000b). Freezing by heating in a driven mesoscopic system. Phys. Rev. Lett. 84: 1240-1243. URL http://link.aps.org/doi/10.1103/PhysRevLett.84.1240.

Helbing, D. and Huberman, B. A. (1998). Coherent moving states in highway traffic. Nature 396: 738-740. URL http://dx.doi.org/10.1038/25499.

Helbing, D. And Lämmer, S. (2005). Supply and production networks: from the bullwhip effect to business cycles. In Networks of Interacting Machines: Production Organization in Complex Industrial Systems and Biological Cells, D. Armbruster, A. S. Mikhailov, and K. Kaneko, (Eds.). World Scientific, Singapore, 33-66.

Helbing, D. And Mazloumian, A. (2009). Operation regimes and sloweris-faster effect in the controlof traffic intersections. The European Physical Journal B - Condensed Matter and Complex Systems 70: 257-274. URL http://dx.doi.org/10.1140/epjb/e2009-00213-5.

Helbing, D. And Molnár, P. (1995). Social force model for pedestrian dynamics. Physical review E 51 (5): 4282.

Helbing, D. And Nagel, K. (2004). The physics of traffic and regional development. Contemporary Physics 45 (5): 405-426. URL http: //www. tandfonline.com/doi/abs/10.1080/00107510410001715944.

Helbing, D., Seidel, T., LÄmmer, S., And Peters, K. (2006). Self-organization principles in supply networks and production systems. In Econophysics and Sociophysics, B. K. Chakrabarti, A. Chakraborti, and A. Chatterjee, (Eds.). Wiley, Weinheim, 535-559. URL http://dx.doi.org/10.1002/9783527610006.ch19. 
Helbing, D. And Treiber, M. (1998). Jams, waves, and clusters. Science 282 (5396): 2001-2003. URL http://arxiv.org/abs/cond-mat/9812299.

Heliövaara, S., Ehtamo, H., Helbing, D., and Korhonen, T. (2013). Patient and impatient pedestrians in a spatial game for egress congestion. Phys. Rev. E 87: 012802. URL http://link.aps.org/doi/10.1103/PhysRevE.87.012802.

Jiang, R., Helbing, D., Shukla, P. K., And Wu, Q.-S. (2006). Inefficient emergent oscillations in intersecting driven many-particle flows. Physica A: Statistical Mechanics and its Applications 368 (2): 567 - 574. URL http://www.sciencedirect.com/science/article/pii/S0378437106000070.

Kesting, A., Treiber, M., And Helbing, D. (2007). General lane-changing model MOBIL for car-following models. Transportation Research Record: Journal of the Transportation Research Board 1999 (1): 86-94.

Kirchner, A., Klüpfel, H., Nishinari, K., Schadschneider, A., AND SchreckenberG, M. (2003). Simulation of competitive egress behavior: comparison with aircraft evacuation data. Physica A: Statistical Mechanics and its Applications 324 (3-4): 689 - 697. URL http://www.sciencedirect.com/science/article/pii/S0378437103000761.

LÄmmer, S. AND Helbing, D. (2008). Self-control of traffic lights and vehicle flows in urban road networks. J. Stat. Mech. 2008: P04019. URL http://dx.doi.org/10.1088/1742-5468/2008/04/P04019.

LÄmmer, S. And Helbing, D. (2010). Self-stabilizing decentralized signal control of realistic, saturated network traffic. Tech. Rep. 10-09-019, Santa Fe Institute.

LÄmmer, S., Kori, H., Peters, K., And Helbing, D. (2006). Decentralised control of material or traffic flows in networks using phase-synchronisation. Physica A 363 (1) (April): 39-47. URL http://dx.doi.org/10.1016/j.physa.2006.01.047.

Maunder, M. N. (2002). The relationship between fishing methods, fisheries management and the estimation of maximum sustainable yield. Fish and Fisheries 3 (4): 251-260. URL http://dx.doi.org/10.1046/j.1467-2979.2002.00089.x.

NarAng, R. K. (2013). High-speed trading. In Inside the Black Box: A Simple Guide to Quantitative and High-Frequency Trading, 2nd ed. John Wiley \& Sons, Inc., Hoboken, NJ, USA, 243-264. URL http://dx.doi.org/10.1002/9781118662717.ch14.

Pauly, D., Christensen, V., Dalsgand, J., Froese, R., And Torres, F. (1998). Fishing down marine food webs. Science 279 (5352): 860-863. URL http://dx.doi.org/10.1126/science.279.5352.860.

Peters, K., Seidel, T., LÄmmer, S., And Helbing, D. (2008). Logistics networks: Coping with nonlinearity and complexity. In Managing Complexity: Insights, Concepts, Applications, D. Helbing, (Ed.). Springer Berlin Heidelberg, 119-136. URL http://dx.doi.org/10.1007/978-3-540-75261-5_6. 
Russell, S. And Norvig, P. (2003). Artificial intelligence: a modern approach, 2nd ed. Prentice Hall, Upper Saddle River, New Jersey.

Sachs, J. L., Mueller, U. G., Wilcox, T. P., And Bull, J. J. (2004). The evolution of cooperation. The Quarterly Review of Biology 79 (2): pp. 135-160. URL http://www.jstor.org/stable/10.1086/383541.

Seidel, T., Hartwig, J., Sanders, R. L., And Helbing, D. (2008). An agentbased approach to self-organized production. In Swarm Intelligence: Introduction and Applications, C. Blum and D. Merkle, (Eds.). Springer, Berlin, 219-252. URL http://dx.doi.org/10.1007/978-3-540-74089-6_7.

Sellis, D., Callahan, B. J., Petrov, D. A., And Messer, P. W. (2011). Heterozygote advantage as a natural consequence of adaptation in diploids. Proceedings of the National Academy of Sciences 108 (51): 20666-20671. URL http: //www . pnas.org/content/108/51/20666. abstract.

Siegel, A. N. (2013). Combinatorial Game Theory. American Mathematical Society.

Slobodkin, L. B. (1961). Growth and Regulation of Animal Populations. Holt, Reinhart and Winston, New York.

Stanley, H. E. (2000). Non-equilibrium physics: Freezing by heating. Nature 404 (6779) (04): 718-719. URL http://dx.doi.org/10.1038/35008188.

Stark, H.-U., Tessone, C. J., And Schweitzer, F. (2008a). Decelerating microdynamics can accelerate macrodynamics in the voter model. Phys. Rev. Lett. 101: 018701. URL http://link.aps.org/doi/10.1103/PhysRevLett.101.018701.

Stark, H.-U., Tessone, C. J., And Schweitzer, F. (2008b). Slower is faster: Fostering consensus formation by heterogeneous inertia. Advances in Complex Systems 11 (04): $551-563$.

Steinberg, R. and Zangwill, W. I. (1983). The prevalence of Braess' paradox. Transportation Science 17 (3): 301-318. URL http://dx.doi.org/10.1287/trsc.17.3.301.

Toppe, J., Bondad-Reantaso, M., Hasan, M., Josupeit, H., Subasinghe, R., HalWART, M., AND JAMES, D. (2012). Aquatic biodiversity for sustainable diets: the role of aquatic foods in food and nutrition security. In Sustainable diets and biodiversity: directions and solutions for policy, research and action, B. Burlingame and S. Dernini, (Eds.). FAO, Rome, 94-101. URL http://www.fao.org/docrep/016/i3004e/i3004e.pdf.

Vilone, D., Vespignani, A., And Castellano, C. (2002). Ordering phase transition in the one-dimensional axelrod model. The European Physical Journal B - Condensed Matter and Complex Systems 30 (3): 399-406. URL http://dx.doi.org/10.1140/epjb/e2002-00395-2. 
Virgo, N., Froese, T., And Ikegami, T. (2013). The positive role of parasites in the origins of life. In Artificial Life (ALIFE), 2013 IEEE Symposium on. IEEE, pp. 1-4. URL http://dx.doi.org/10.1109/ALIFE.2013.6602424.

Watson, R. A., Mills, R., And Buckley, C. L. (2011). Global adaptation in networks of selfish components: Emergent associative memory at the system scale. Artificial Life 17 (3) (2015/06/01): 147-166. URL http://dx.doi.org/10.1162/art1_a_00029.

Wolfram, S. (2002). A New Kind of Sciene. Wolfram Media. URL http://www.wolframscience.com/.

Wolpert, D. H. and Macready, W. G. (1995). No free lunch theorems for search. Tech. Rep. SFI-WP-95-02-010, Santa Fe Institute. URL http://tinyurl.com/yz274ej.

Wolpert, D. H. and Macready, W. G. (1997). No Free Lunch Theorems for Optimization. IEEE Transactions on Evolutionary Computation 1 (1): 67-82.

Zubillaga, D., Cruz, G., Aguilar, L. D., Zapotécatl, J., Fernández, N., Aguilar, J., Rosenblueth, D. A., and Gershenson, C. (2014). Measuring the complexity of self-organizing traffic lights. Entropy 16 (5): 2384-2407. URL http://dx.doi.org/10.3390/e16052384. 